\title{
Addition of 25-hydroxyvitamin D levels to the Deyo-Charlson Comorbidity Index improves 90 -day mortality prediction in critically ill patients
}

Bisundev Mahato ${ }^{1,2,3}$, Tiffany M. N. Otero ${ }^{1,4}$, Carrie A. Holland ${ }^{5}$, Patrick T. Giguere ${ }^{1}$, Ednan K. Bajwa ${ }^{5,7}$, Carlos A. Camargo Jr. ${ }^{6,7,8}$ and Sadeq A. Quraishi ${ }^{1,9^{*}}$

\begin{abstract}
Background: The Deyo-Charlson Comorbidity Index (DCCI) has low predictive value in the intensive care unit (ICU). Our goal was to determine whether addition of 25-hydroxyvitamin D (25OHD) levels to the DCCl improved 90-day mortality prediction in critically ill patients.

Methods: Plasma 25OHD levels, DCCl, and Acute Physiology and Chronic Health Evaluation II (APACHE II) scores were assessed within $24 \mathrm{~h}$ of admission in 310 ICU patients. Receiver operating characteristic curves of the prediction scores, without and with the addition of $250 \mathrm{HD}$ levels, for 90 -day mortality were constructed and the areas under the curve (AUC) were compared for equality.

Results: Mean (standard deviation) plasma 25OHD levels, DCCl, and APACHE II score were 19 (SD 8) ng/mL, 4 (SD 3), and 17 (SD 9), respectively. Overall 90-day mortality was $19 \%$. AUC for DCCl vs. DCCl $+250 \mathrm{HD}$ was 0.68 (95\% Cl $0.58-0.77$ ) vs. 0.75 (95\% Cl 0.67-0.83); $p<0.001$. AUC for APACHE ॥ vs. APACHE $\|+25 \mathrm{OHD}$ was $0.81(95 \% \mathrm{Cl}$ $0.73-0.88$ ) vs. 0.82 ( $95 \% \mathrm{Cl} 0.75-0.89$ ); $p<0.001$. There was a significant difference between the AUC for $\mathrm{DCCl}+25 \mathrm{OHD}$ and APACHE $\|+25 \mathrm{OHD}(p=0.04)$ but not between the AUC for DCCI $+25 \mathrm{OHD}$ and APACHE $\|(p=0.12)$.

Conclusions: In our cohort of ICU patients, the addition of 25OHD levels to the DCCI improved 90-day mortality prediction compared to the $\mathrm{DCCl}$ alone. Moreover, the predictive capability of $\mathrm{DCCl}+25 \mathrm{OHD}$ was comparable to that of APACHE II. Future prospective studies are needed to validate our findings and to determine whether the use of $\mathrm{DCCl}+25 \mathrm{OHD}$ can influence clinical decision-making.
\end{abstract}

Keywords: Vitamin D, 25-hydroxyvitamin D, Mortality, ICU

Abbreviations: 25OHD, 25-hydroxyvitamin D; APACHE II, Acute physiology and Chronic Health Evaluation score II; AUC, area under the curve; BMI, body mass index; DCCI, The Deyo-Charlson Comorbidity Index; EMR, Electronic medical record; ICU, intensive care unit; LOWESS, locally weighted scatterplot smoothing; MGH, Massachusetts General Hospital; OR, odds ratio; POC, point of care; ROC, receiver operating curve; SD, standard deviation; STATA, StataCorp LP; VDSP, Vitamin D Standardization Program

\footnotetext{
* Correspondence: squraishi@mgh.harvard.edu

${ }^{1}$ Department of Anesthesia, Critical Care and Pain Medicine, Massachusetts

General Hospital, 55 Fruit Street, GRJ 402, Boston, MA, USA

${ }^{9}$ Department of Anaesthesia, Harvard Medical School, Boston MA, USA

Full list of author information is available at the end of the article
} 


\section{Background}

Mortality prediction scores are widely used in the intensive care unit (ICU) to predict the likelihood of survival from critical illness. These scores often assist healthcare providers in their discussions with patients and their family members about realistic expectations regarding ICU care and may be helpful in setting more patient-centered goals, assessing resource utilization, and providing higher quality of care [1]. Mortality prediction scores are also widely used by researchers to risk adjust for severity of illness, especially in multivariable regression models $[2,3]$.

While several mortality prediction scores for critical illness have been developed and are in current use, the Acute Physiology and Chronic Health Evaluation II (APACHE II), first published in 1985 [4], is the most commonly used worldwide [5]. However, APACHE II score calculations require measurement of specific physiological parameters within $24 \mathrm{~h}$ of ICU admission-this can be challenging, especially if a robust electronic medical record system is not available. Moreover, given that the score is heavily based on post-admission physiological assessments, it is not readily available at the time of admission, and it is of limited value for decision-making at the outset of critical illness.

Given the challenges of the APACHE II calculation (and for other similar mortality prediction models heavily influenced by physiologic assessments), the use of scoring systems based largely on medical history (i.e., comorbidities), despite being less accurate, are growing in popularity in the ICU literature [6, 7]. Among these, the Deyo-Charlson Comorbidity Index (DCCI) has been reported most frequently in studies of critical illness [8]. The DCCI [9], although modified from the original Charlson Index [6], continues to have a low predictive value for mortality in ICU patient cohorts [10-14]. Various modifications of either the original Charlson Index or DCCI have been made to improve their predictive values. These modifications have typically involved the use of administrative data [15-18], and they are not specific to ICU patients. And although the inclusion of several laboratory test results in the APACHE II scoring method is integral to its superior performance over the DCCI for mortality prediction in ICU patients [19], the impact of adding easily measurable or readily available biomarkers to improve the predictive capabilities of the DCCI in ICU patients has been largely underexplored [20].

Mounting evidence suggests that vitamin D status is associated with mortality in critically ill patients [21-27]. Indeed, assessment of vitamin D status in ICU patients is becoming increasingly common in the USA and worldwide $[21,28,29]$. In general, serum 25 -hydroxyvitamin D (25OHD) levels are considered the best marker of total body vitamin D status [30]. Therefore, our goal was to determine whether the addition of 25OHD levels, measured at the outset of critical illness, to the DCCI improves mortality prediction in ICU patients.

\section{Methods}

We performed a retrospective analysis of the data from an ongoing prospective cohort study designed to assess vitamin D status in critically ill patients. A subset of these patients was previously described in studies that investigated the association of vitamin D status with duration of mechanical ventilation and 90-day mortality in ICU patients $[25,31]$. For the present study, subjects were recruited from three, 18-bed ICUs (1 surgical, 1 medical, and 1 mixed surgical/medical) at the Massachusetts General Hospital (MGH), in Boston, MA. The ICUs received admissions from all surgical and medical services except for Cardiac Surgery and Cardiology, respectively. All subjects were enrolled between 06/01/2012 and 05/30/2015. MGH is a 1052-bed, teaching hospital and a level-one trauma center, which serves a diverse population in and around Eastern Massachusetts. The Partners Human Research Committee (Institutional Review Board) approved the study protocol.

\section{Inclusion and exclusion criteria}

All adult males and females, $\geq 18$ years of age, and who were expected to require at least $48 \mathrm{~h}$ of critical care (as determined by the treating ICU team) were deemed eligible to participate. Informed consent was obtained either directly from subjects or appropriate healthcare surrogates. Subjects were only included in the study if blood samples to assess vitamin D status could be obtained within $24 \mathrm{~h}$ of admission to the ICU. Exclusion criteria included a known history of anemia at the time of ICU admission (defined as hematocrit $<25 \%$ ), pregnancy or immediate post-partum status, and history of vitamin D supplementation $\geq 4000 \mathrm{IU} /$ day. To minimize confounding from either partially treated, new-onset illness, or chronic illnesses, subjects were also excluded if they were transferred from another ICU or had been in an ICU within 1 year of the most current admission. Patients expected to transition to "comfort only measures" were also excluded.

\section{Blood sample processing and biomarker assays}

Following informed consent, fresh blood was acquired from an indwelling arterial or central venous catheter and was collected directly into an EDTA-containing tube (lavender top). The sample was immediately stored on ice and then centrifuged within $30 \mathrm{~min}$ to separate out plasma. All samples were centrifuged at $2300 \mathrm{rpm}$ for $15 \mathrm{~min}$ at a temperature of $4{ }^{\circ} \mathrm{C}$. The separated plasma was immediately transferred to polypropylene tubes and stored at $-80{ }^{\circ} \mathrm{C}$ until biomarker testing was ready to be 
initiated. Assays were performed at the Harvard Medical School Clinical and Translational Science Award core laboratory at MGH. Plasma 25OHD (combined $\mathrm{D}_{2}$ and $\mathrm{D}_{3}$ ) levels were measured by enzyme-linked immunoabsorbent assay, using commercially available kits (Abbott Laboratories, Abbott Park, IL). Intra- and inter-assay coefficients of variation were both $<10 \%$.

\section{Clinical data collection}

The MGH electronic medical records system used abstract baseline demographic information, including (1) age, (2) sex, (3) race, (4) body mass index (BMI), and (5) type of patient (surgical vs. medical). To obtain inhospital mortality data within 90-days of ICU admission, individual electronic medical records were reviewed. For patients discharged alive from the hospital and who continued to receive care within the Partners Healthcare network (which includes MGH and its affiliates), individual electronic outpatient medical records were reviewed to document all-cause mortality within 90 days of ICU admission. All individual records were cross-referenced with the Social Security Death Index Master File to finalize 90-day mortality cases.

\section{Statistical analysis}

Descriptive statistics were tabulated for the analytic cohort. Continuous data were reported as means with standard deviations (SDs), and categorical values were expressed as proportions. To graphically represent the relationship between 25OHD levels and DCCI as well as APACHE II, we constructed locally weighted scatterplot smoothing (LOWESS) curves. LOWESS is a type of nonparametric regression, which summarizes a relationship between two variables in a fashion that initially relies on limited assumptions about the form or strength of the relationship [32]. The rationale and methods underlying the use of LOWESS for depicting the local relationship between measurements of interest across parts of their ranges have previously been described [33]. To investigate the association of 25OHD levels with 90-day mortality, we performed logistic regression analyses while controlling for biologically plausible covariates-as such, we developed two main models: the first controlled for (1) age, (2) sex, (3) race, (4) BMI, (5) type of patient, and (6) DCCI, while the second controlled for (1) age, (2) sex, (3) race, (4) BMI, (5) type of patient, and (6) APACHE II score.

Receiver operating characteristic (ROC) curves were constructed to assess the areas under the curve (AUC) for the ability of each predictive model to correctly identify survivors vs. non-survivors. An AUC of 0.5 suggests no predictive value, $0.7-0.8$ suggests good predictive value, and $>0.8$ suggests excellent predictive value. Four different models were tested for their ability to predict 90 - day mortality in the analytic cohort, namely (1) DCCI, (2) $\mathrm{DCCI}+25 \mathrm{OHD}$, (3) APACHE II, and (4) APACHE II + 25OHD. 25OHD levels were categorized into nationally accepted threshold levels of $<10 \mathrm{ng} / \mathrm{mL}, 10-19.9 \mathrm{ng} / \mathrm{mL}$, 20-29.9 ng/mL, and $\geq 30 \mathrm{ng} / \mathrm{mL}$. Recent evidence suggests that these thresholds may be relevant and applicable to hospitalized and critically ill patients as well [34-36]. We assigned all plausible serial permutations of the sub-score values of the DCCI and APACHE II (0-6) to each 25OHD category in order to derive the highest AUC value. Serial number scores were assigned since the existing literature suggests that there is a near inverse linear association between 25OHD levels and adverse outcomes in hospitalized patients [34-36]. The final AUC values were compared for equality using the methodology described elsewhere [37]. Moreover, optimal cut-points were determined from the ROC curves by identifying scores that provided the highest cumulative value for sensitivity and specificity.

In a previous study [25], our group demonstrated that 100 ICU patients provided sufficient power to detect a meaningful association between admission 25OHD level as well as APACHE II score and 90-day mortality. Therefore, we assumed that the current analytic cohort size ( $n=310$ ) would be adequately powered to undertake the study objectives. All analyses were performed in STATA 13.0 (StataCorp LP, College Station, TX). A two-tailed $p<0.05$, and any $95 \%$ confidence interval not spanning 1 , was considered statistically significant for all analyses.

\section{Results}

Three hundred and ten patients comprised the analytic cohort. All blood samples were obtained within $24 \mathrm{~h}$ of ICU admission, with $72 \%$ of samples being obtained within $6 \mathrm{~h}$ of admission to the ICU. The general characteristics of the study cohort, stratified by 90-day survivors vs. non-survivors, are shown in Table 1. LOWESS curve analysis demonstrated a near inverse relationship between DCCI as well as APACHE II and 25OHD levels between 0 and $10 \mathrm{ng} / \mathrm{mL}$ (Fig. 1). Between 25OHD levels of 10 and $30 \mathrm{ng} / \mathrm{mL}$, there was significant flattening of the curve. Multivariable logistic regression analyses including either DCCI or APACHE II score as a covariate demonstrated an inverse association between 25OHD levels and 90-day mortality in both models (OR 0.84; $95 \%$ CI $0.79-0.91$ and OR 0.86; 95 \% CI 0.80-0.93, per $1 \mathrm{ng} / \mathrm{mL}$, respectively). DCCI was independently associated with 90-day mortality (OR 1.32; $95 \%$ CI 1.12-1.57), as was APACHE II (OR 1.14; 95 \% CI 1.08-1.21).

Initial score assignment for $25 \mathrm{OHD}$ thresholds was 0 for levels $\geq 30 \mathrm{ng} / \mathrm{mL}$ (since this is widely regarded as a "normal" level), 1 for levels $20-29.9 \mathrm{ng} / \mathrm{mL}, 2$ for levels 10-19.9 ng/mL, and 3 for levels $<10 \mathrm{ng} / \mathrm{mL}$. The respective threshold scores were added to each DCCI and 
Table 1 General characteristics of the study cohort $(n=310)$ stratified by 90-day survivors and non-survivors

\begin{tabular}{|c|c|c|c|}
\hline Variable & $\begin{array}{l}\text { 90-day survivors } \\
(n=251)\end{array}$ & $\begin{array}{l}\text { 90-day non-survivors } \\
(n=59)\end{array}$ & $P$ value \\
\hline Age (years) & $67 \pm 14$ & $69 \pm 18$ & 0.43 \\
\hline Sex (\%) & & & 0.15 \\
\hline Female & 39 & 49 & \\
\hline Male & 61 & 51 & \\
\hline Race (\%) & & & $<0.001$ \\
\hline Non-white & 5 & 32 & \\
\hline White & 95 & 68 & \\
\hline BMI $\left(\mathrm{kg} / \mathrm{m}^{2}\right)$ & $28 \pm 7$ & $30 \pm 8$ & 0.08 \\
\hline Type of patient (\%) & & & $<0.001$ \\
\hline Surgical & 76 & 43 & \\
\hline Medical & 24 & 57 & \\
\hline $250 \mathrm{HD}(\mathrm{ng} / \mathrm{mL})$ & $20 \pm 14$ & $12 \pm 7$ & $<0.001$ \\
\hline APACHE ॥ & $14 \pm 7$ & $24 \pm 9$ & $<0.001$ \\
\hline Comorbidities (\%) & & & 0.21 \\
\hline Cardiovascular & 92 & 97 & \\
\hline Pulmonary & 24 & 46 & \\
\hline Renal & 35 & 44 & \\
\hline Hepatic & 5 & 12 & \\
\hline Sepsis & 38 & 51 & \\
\hline Trauma & 20 & 36 & \\
\hline $\mathrm{DCCl}$ & $3 \pm 2$ & $5 \pm 3$ & $<0.001$ \\
\hline
\end{tabular}

Continuous data were reported as means with standard deviations (SDs), medians with interquartile ranges (IQRs), and categorical values were expressed as proportions. Body mass index $=$ BMI, Acute Physiology and Chronic Health Evaluation II= APACHE II; Deyo-Charlson Comorbidity Index $=\mathrm{DCCl}$; intensive care unit $=\mathrm{ICU}$

$\mathrm{P}$-values in italics represent statistically significant results
APACHE II score and ROC curves were generated to assess the AUCs (Fig. 2). Alternative score assignments did not result in either a statistically different, clinically meaningful, or biologically plausible difference in the calculated AUCs for DCCI + 25OHD or APACHE II + 25OHD. Moreover, based on the observed relationship between 25OHD and DCCI as well as APACHE II on LOWESS curve analysis, we also generated models where $25 \mathrm{OHD}$ levels $\geq 30 \mathrm{ng} / \mathrm{mL}$ designated a score of 0 , levels $10-29.9 \mathrm{ng} / \mathrm{mL}$ were designated a score of 1 , and levels $<10 \mathrm{ng} / \mathrm{mL}$ were designated a score of 3. Again, this alternative scoring designation did not materially change any previously obtained results. As such, for the final models, the initial scoring assignment was retained. AUC for 25OHD alone was 0.75 (95\% CI 0.67-0.83). On the other hand, AUC for DCCI alone was 0.68 (95\% CI 0.59-0.77), and improved to 0.75 (95\% CI 0.66-0.83) with the addition of 25OHD threshold scores $\left(X^{2}=19.1\right.$, $p<0.001$ ). AUC for APACHE II alone was 0.81 (95\% CI $0.73-0.88$ ) and increased slightly to 0.82 (95 \% CI $0.75-$ 0.89 ) with the addition of $25 \mathrm{OHD}$ threshold scores $\left(X^{2}=11.4, p<0.001\right)$. There was no difference between the AUC for 25OHD alone vs. DCCI + 25OHD $\left(X^{2}=0, p=0.97\right)$. And while there was a significant difference between the AUC for DCCI + 25OHD vs. APACHE II + 25OHD $\left(X^{2}=4.4, p=0.04\right)$, there was no difference in the AUC for $\mathrm{DCCI}+25 \mathrm{OHD}$ vs. APACHE II alone $\left(X^{2}=2.38, p=0.12\right)$.

The optimal cutoff value for $25 \mathrm{OHD}$ alone was $\geq 3$ (i.e., $<10 \mathrm{ng} / \mathrm{mL}$ ), with a sensitivity of $45 \%$, specificity of $91 \%$, and accuracy of $68 \%$, while the optimal cutoff value for DCCI alone was $\geq 4$, with a sensitivity of $63 \%$, specificity of $63 \%$, and accuracy of $63 \%$. On the other hand, the optimal cutoff value for DCCI $+25 \mathrm{OHD}$ was $\geq 5$, with a sensitivity of $84 \%$, specificity of $55 \%$, and accuracy of $70 \%$. The optimal cutoff value for APACHE II was $\geq 12$, with a sensitivity of $95 \%$, specificity of $42 \%$,
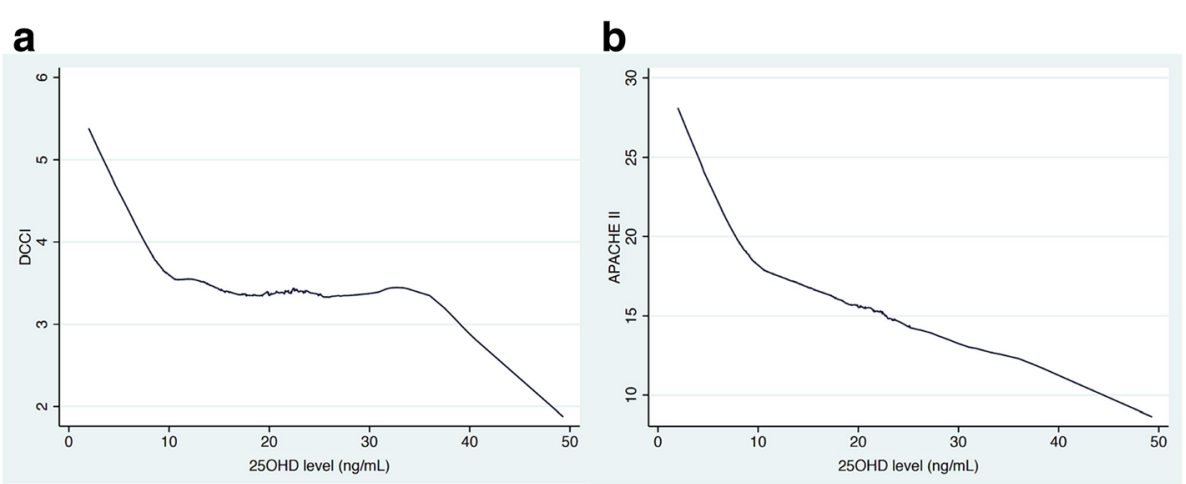

Fig. 1 Locally weighted scatterplot smoothing (LOWESS) curve analysis of the relationship between vitamin D status and severity of illness scores. $\mathrm{DCCl}=$ Deyo-Charlson Comorbidity Index; $25 \mathrm{OHD}=25$-hydroxyvitamin D; APACHE II = Acute Physiology and Chronic Health Evaluation II. LOWESS curve analysis demonstrates a steep, near inverse, relationship between DCCI (a) as well as APACHE II (b) and $250 \mathrm{HD}$ levels from 0 to $10 \mathrm{ng} / \mathrm{mL}$. Beyond $25 \mathrm{OHD}$ levels of 10 , there is progressive flattening of the curve 


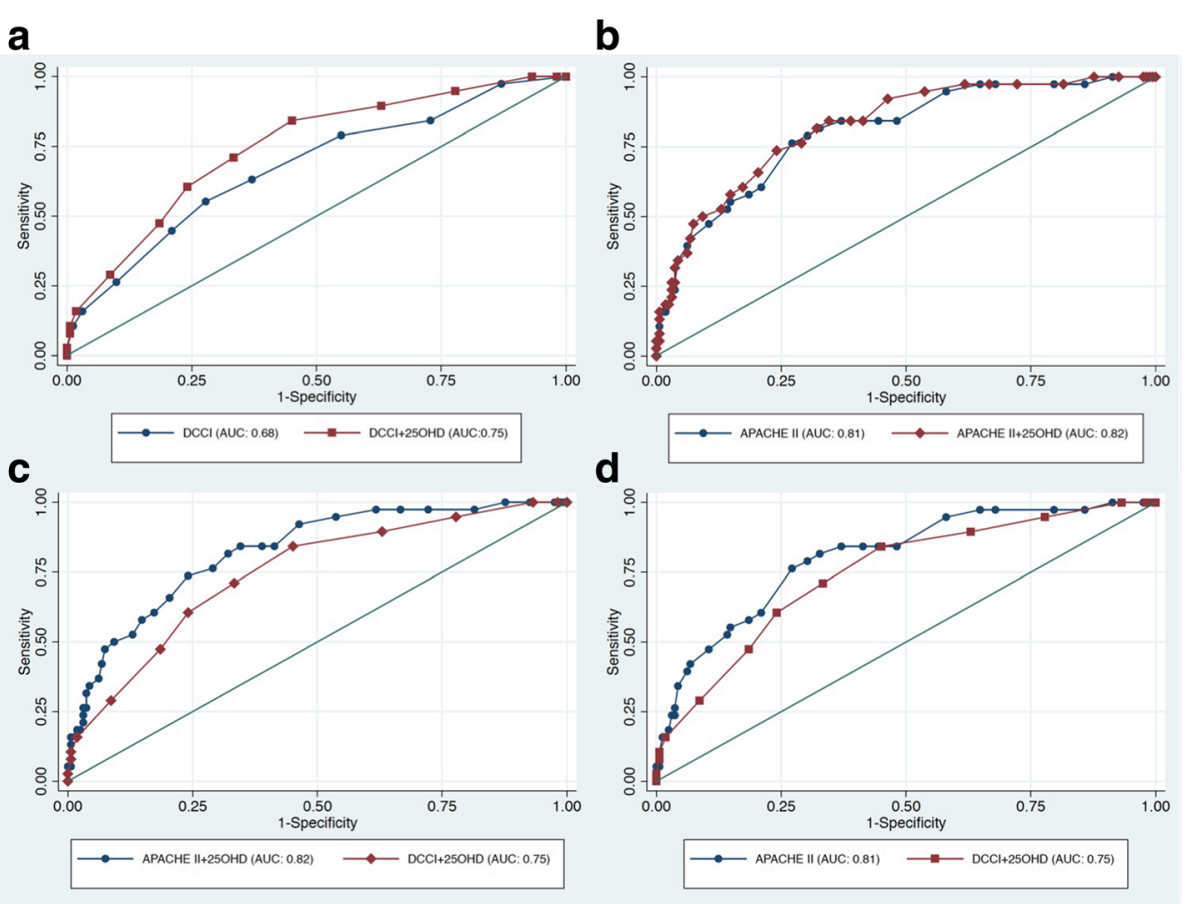

Fig. 2 Receiver operating characteristic (ROC) curves and area under the curves (AUCs). DCCl= Deyo-Charlson Comorbidity Index; 25OHD=25hydroxyvitamin D; APACHE $\|=$ Acute Physiology and Chronic Health Evaluation II. a DCCI vs. DCCI $+25 \mathrm{OHD}(p<0.001)$. b APACHE II vs. APACHE $\|+25 \mathrm{OHD}(p<0.001) \cdot \mathbf{c}$ APACHE $\|+25 \mathrm{OHD}$ vs. $\mathrm{DCCl}+25 \mathrm{OHD}(p=0.12) \cdot \mathbf{d} \mathrm{APACHE} \| \mathrm{vs} . \mathrm{DCCl}+25 \mathrm{OHD}(p=0.04)$

and accuracy of $69 \%$, while the optimal cutoff value for APACHE II $+25 \mathrm{OHD}$ was $\geq 15$, with a sensitivity of $92 \%$, specificity of $54 \%$, and accuracy of $73 \%$.

\section{Discussion}

In this retrospective, cohort study, we investigated whether the addition of 25OHD levels to the DCCI at initiation of care improved 90-day mortality prediction (vs. the DCCI alone) in ICU patients and we compared these findings to the gold standard of APACHE II assessments. We demonstrated that the addition of 25OHD levels significantly improved the AUC for the DCCI, but did not materially affect the predictive capability of the APACHE II. Moreover, our results suggest that the DCCI $+25 \mathrm{OHD}$ model may perform as well as the APACHE II alone for predicting 90-day mortality in ICU patients. However, given the retrospective nature of our study, the real-time utility of DCCI $+25 \mathrm{OHD}$ over the APACHE II requires further investigation.

Previous studies have reported AUCs for the APACHE II method, which demonstrates excellent discrimination between survivors and non-survivors of critical illness (AUC >0.80) [4, 38, 39]. AUC for the APACHE II ROC in our study is consistent with what has been reported in the existing literature. Despite its clinically acceptable predictive value, APACHE II score calculations can be cumbersome if an appropriate infrastructure is not in place. Although most commercially available electronic medical record (EMR) systems automatically calculate the APACHE II score, these products may be costprohibitive, especially for smaller healthcare entities [40]. And without a robust EMR, APACHE II calculations must be performed manually by healthcare providers, which can be time consuming and therefore prone to low compliance. Furthermore, APACHE II scores may provide prognostic information too late after ICU admission. Since patients need to be admitted to the ICU for at least $24 \mathrm{~h}$ before a score can be calculated, the APACHE II does not help with critical decision-making for patients and families at the outset of critical illness. Indeed, many patients and families struggle with decisions to prolong medical therapy and/or undergo potentially painful as well as invasive procedures on admission to the ICU. As such, simple and timely predictive models for ICU-related mortality have the potential to assist clinicians in delivering more patient-centered, higher quality, and cost-effective care.

In 1987, Charlson et al. developed a weighted index, which took into account both the number and severity of various comorbid diseases; the purpose of the index was to develop a 10-year mortality prediction tool for the general population [6]. In its original form, the Charlson Comorbidity Index relied on patient interviews or chart review to classify comorbid diseases. Later, Deyo et al. adapted it to accommodate the use of the International Classification of Disease, 9th Edition, 
Clinical Modification (ICD-9-CM) to calculate the DCCI from administrative databases [9]. Other modifications to the original Charlson Comorbidity Index have included the use of alternate lists of specific diagnoses for comorbidities [15], re-assigning comorbidity weights [16, 17], and updating to the ICD-10-CM codes from ICD-9-CM codes $[16,18]$. Recently, an increasing number of publications in acute care medicine have used the DCCI to riskadjust for severity of illness [34-36, 41, 42]-indeed, mounting evidence suggests that the DCCI may have excellent in-hospital, 30-day, and 1-year mortality prediction in hospitalized patients [43]. Regarding the ICU, DCCI alone appears to have low predictive value (which is also observed in the present study) [41], but in combination with administrative data that reflect acute physiological processes (e.g., admission diagnosis, need for mechanical ventilation, initiation of renal replacement therapy), the AUC of the modified DCCI may be equivalent to the APACHE II [44]. While such adjustments are helpful for research purposes, they do not apply to real-time decision-making at the outset of critical illness. The use of non-administrative data, such as readily available or easily measured biomarkers, in conjunction with the DCCI has been reported to improve mortality prediction in dialysisdependent renal failure patients [20] but until now has largely remained unexplored in the ICU.

Our study suggests that using easily obtained biomarker data, more specifically 25OHD levels at initiation of care, may significantly improve the predictive value of the DCCI for 90-day mortality in ICU patients, potentially making it a feasible alternative to the APACHE II score, especially when the necessary acute physiologic assessments are not readily available. Indeed, several studies suggest that 25OHD levels at initiation of critical care are inversely associated with mortality $[25,29,45]$. While vitamin D has traditionally been thought to play a vital role in bone [46], cardiac [47, 48], and muscle health [49], primarily by maintaining calcium balance, recent data support a more pleiotropic effect of vitamin $\mathrm{D}$ on general health [46]. With regards to critical illness, vitamin D plays a major role in maintaining immune heath. Recent studies have demonstrated that cells of the innate and adaptive immune system express the vitamin D receptor. Low $25 \mathrm{OHD}$ levels are associated with depressed macrophage phagocytosis, attenuated chemotaxis, and proinflammatory cytokine production [50]. Macrophages activated through the vitamin $\mathrm{D}$ receptor by 1,25 dihydroxyvitamin $\mathrm{D}$ (the most hormonally active vitamin D metabolite) upregulate expression of cathelicidin [51]. Cathelicidins are endogenous antimicrobial peptides that are active against a broad spectrum of infectious agents, such as bacteria, viruses, fungi, and mycobacteria [52]. Moreover, cathelicidins are highly expressed by epithelial cells at natural barrier sites (e.g., skin, lungs, gut) and may represent an important first line of defense for the innate immune system [53]. In addition, vitamin $\mathrm{D}$ is important for the interferon- $\gamma$-dependent $\mathrm{T}$ cell responses to infection [54] and therefore may play an important role in preventing immunoparalysis [55]. And finally, in animal models, vitamin D supplementation has been shown to improve coagulation variables and inhibit endotoxemia [56-58].

It is important to note that at present, it is not standard practice in most institutions worldwide to routinely obtain 25OHD levels in ICU patients. Traditionally, this has been due to a lack of evidence suggesting that assessment of vitamin D status might be helpful in critically ill patients; however, recent evidence supports the notion that 25OHD may be an important biomarker in this patient cohort [30, 35, 36]. Other major barriers to readily assessing vitamin $\mathrm{D}$ status in hospitalized patients worldwide have been the lack of in-house laboratory equipment, batched processing protocols, and the cost of the $25 \mathrm{OHD}$ assays themselves. Given the intricacies of accurately measuring steroid hormone levels, many facilities outsource such measurements, and as such, results may take several days to be finalized. Similarly, if the overall volume of testing is insufficient, many facilities process samples at dedicated time intervals-which may present a delay in obtaining results for bedside clinical decisionmaking. Led by the US National Institute of Standards and Technology and the National Institutes of Health, a global Vitamin D Standardization Program (VDSP) has been launched to assist facilities in ensuring the quality of their in-house assays and results [59-62]. Moreover, the recent availability of various point-of-care (POC) vitamin $\mathrm{D}$ testing devices has now made it feasible to obtain 25OHD levels to allow for real-time, bedside clinical decision-making [63-65]. These devices require very small amounts of blood (some are similar in design to POC glucose testing units for diabetics), are very affordable, and provide results within $10 \mathrm{~min}$.

Although we present intriguing evidence regarding the addition of $25 \mathrm{OHD}$ and the DCCI to significantly improve mortality prediction in ICU patients, it is important to be mindful of potential limitations of our work. Retrospective cohort studies such as this cannot establish causation, but it can highlight the existence or absence of associations and thereby direct future research. Furthermore, observational studies may be limited by the lack of a randomly distributed exposure. And despite adjustment for the multiple potential confounders within the DCCI and APACHE II, there may still be residual confounding that contributes to the observed differences in survival. More specifically, vitamin D status may simply be a reflection of the overall health of patients, for which we may be unable to fully adjust. We were also unable to adjust for immobilization, lack of sun exposure, and 
vitamin D supplementation over the study period. In addition, 25OHD was only assessed within $24 \mathrm{~h}$ of ICU admission and may not completely represent vitamin D status at the outset of critical illness (since 25OHD levels may be influenced by inflammatory responses, fluid loading, and renal wasting of albumin as well as vitamin D binding protein) [30]. Moreover, changes in vitamin D status over the course of the study period were not assessed. The results of our study also may not be generalizable, since patients from only one institution and from only surgical or medical ICUs were included in the analysis (i.e., excluded patients from the neurosciences, cardiac, and burn ICUs). We also had a limited sample size $(n=$ 310), which may further impact the generalizability of our findings. Despite these limitations, the biological plausibility of vitamin D status as a determinant of survival after critical illness is undeniable. Interestingly, 25OHD levels alone had a higher AUC than the DCCI; however, it is unlikely that clinicians would be willing to make key decisions based on a single biomarker and ignore the potential impact of chronic illness on critical care outcomes. Fortunately, the addition of vitamin D status to the DCCI maintains the observed AUC of 25OHD alone and allows for a prediction model that is as accurate as APACHE II alone. As such, the results of our study warrant further investigation to confirm these findings in larger datasets and to conduct future prospective studies.

\section{Conclusions}

We confirm previous studies, which have suggested that total 25OHD levels upon ICU admission are inversely associated with the risk of mortality in the post-acute care setting. In addition, we present novel data that 25OHD levels on admission to the ICU may significantly improve the predictive value of the DCCI for 90-day mortality in critically ill patients. This may be particularly helpful in making clinical decisions at initiation of care in the ICU or in settings where prediction scores heavily based on physiologic assessments may not be readily available. Further prospective studies are needed to validate our findings and to determine whether optimizing vitamin D status in surgical ICU patients may confer survival benefit from critical illness.

\section{Availability of supporting data}

Our Institutional Review Board proposal for this study specifically states that data will not be shared with any party outside of the Partners Healthcare Network; our database will not be available.

\section{Competing interests}

SAQ has received consulting fees from Lungpacer, Inc. and Trevena, Inc. In addition to this, SAQ serves as an uncompensated Board member for both the Vitamin D Council and the C. Diff Foundation.

\section{Authors' contributions}

$\mathrm{BM}$ and $\mathrm{SAQ}$ contributed to the concept and design of the manuscript. TMNO and CAH contributed to the acquisition and interpretation of the data. BM, TMNO, and SAQ drafted the manuscript. TMNO, PTG, EKB, and CAC $J$ r. revised the manuscript. All authors agree to be fully accountable for ensuring the integrity and accuracy of the work and read and approved the final manuscript.

\section{Acknowledgements}

Not applicable

\section{Financial disclosures}

TMNO received support from the National Center for Advancing Translational Sciences, National Institutes of Health, Award Number TL1TR001062.

CAC received support from the National Institutes of Health grants R01 Al093723 and U01 Al087881. SAQ received support from the National Institutes of Health grants T32 GM007592, UL1 RR025758, L30 GM102903, and L30 TR001257.

\section{Author details}

'Department of Anesthesia, Critical Care and Pain Medicine, Massachusetts General Hospital, 55 Fruit Street, GRJ 402, Boston, MA, USA. ${ }^{2}$ Harvard Medical School, Boston MA, USA. ${ }^{3}$ Department of Anesthesiology \& Perioperative Care, University of California - Irvine, Orange CA, USA. ${ }^{4}$ Tufts University School of Medicine, Boston, MA, USA. ${ }^{5}$ Department of Medicine, Massachusetts General Hospital, Boston, MA, USA. ${ }^{6}$ Department of Emergency Medicine, Massachusetts General Hospital, Boston, MA, USA. ${ }^{7}$ Department of Medicine, Harvard Medical School, Boston, MA, USA.

${ }^{8}$ Department of Epidemiology, Harvard School of Public Health, Boston, MA, USA. ${ }^{9}$ Department of Anaesthesia, Harvard Medical School, Boston MA, USA.

Received: 21 March 2016 Accepted: 15 June 2016

Published online: 17 June 2016

\section{References}

1. Zimmerman JE, Wagner DP, Knaus WA, Williams JF, Kolakowski D, Draper EA. The use of risk predictions to identify candidates for intermediate care units. Implications for intensive care utilization and cost. Chest. 1995;108(2):490-9.

2. Kollef MH, Schuster DP. Predicting intensive care unit outcome with scoring systems. Underlying concepts and principles. Crit. Care Clin. 1994;10(1):1-18.

3. Knaus WA, Wagner DP, Zimmerman JE, Draper EA. Variations in mortality and length of stay in intensive care units. Ann Intern Med. 1993;118(10):753-61.

4. Knaus WA, Draper EA, Wagner DP, Zimmerman JE. APACHE II: a severity of disease classification system. Crit Care Med Oct. 1985;13(10):818-29.

5. Vincent $\mathrm{L}$, Moreno R. Clinical review: scoring systems in the critically ill. Crit Care. 2010;14(2):207.

6. Charlson ME, Pompei P, Ales KL, Mackenzie CR. A new method of classifying prognostic comorbidity in longitudinal studies: development and validation. J Chronic Dis. 1987:40(5):373-83.

7. Elixhauser A, Steiner C, Harris DR, Coffey RM. Comorbidity measures for use with administrative data. Med Care. 1998;36(1):8-27.

8. Sharabiani MT, Aylin P, Bottle A. Systematic review of comorbidity indices for administrative data. Med Care. 2012;50(12):1109-18.

9. Deyo RA, Cherkin DC, Ciol MA. Adapting a clinical comorbidity index for use with ICD-9-CM administrative databases. J Clin Epidemiol. 1992;45(6):613-9.

10. Cleves MA, Sanchez N, Draheim M. Evaluation of two competing methods for calculating Charlson's comorbidity index when analyzing short-term mortality using administrative data. J Clin Epidemiol. 1997;50(8):903-8.

11. Stukenborg GJ, Wagner DP, Connors Jr AF. Comparison of the performance of two comorbidity measures, with and without information from prior hospitalizations. Med Care. 2001;39(7):727-39.

12. Holman CD, Preen DB, Baynham NJ, Finn JC, Semmens JB. A multipurpose comorbidity scoring system performed better than the Charlson index. J Clin Epidemiol. 2005;58(10):1006-14.

13. Li P, Kim MM, Doshi JA. Comparison of the performance of the CMS Hierarchical Condition Category (CMS-HCC) risk adjuster with the Charlson and Elixhauser comorbidity measures in predicting mortality. BMC Health Serv Res. 2010;10:245. 
14. Chu YT, Ng YY, Wu SC. Comparison of different comorbidity measures for use with administrative data in predicting short- and long-term mortality. BMC Health Serv Res. 2010;10:140.

15. Romano PS, Roos LL, Jollis JG. Adapting a clinical comorbidity index for use with ICD-9-CM administrative data: differing perspectives. J Clin Epidemiol. 1993:46(10):1075-9. discussion 1081-1090.

16. Halfon P, Eggli Y, van Melle G, Chevalier J, Wasserfallen JB, Burnand B. Measuring potentially avoidable hospital readmissions. J Clin Epidemiol. 2002;55(6):573-87.

17. Schneeweiss S, Wang PS, Avorn J, Glynn RJ. Improved comorbidity adjustment for predicting mortality in Medicare populations. Health Serv Res Aug. 2003;38(4):1103-20.

18. Quan H, Sundararajan V, Halfon P, Fong A, Burnand B, Luthi JC, Saunders $L D$, Beck CA, Feasby TE, Ghali WA. Coding algorithms for defining comorbidities in ICD-9-CM and ICD-10 administrative data. Med Care. 2005; 43(11):1130-9

19. Poses RM, McClish DK, Smith WR, Bekes C, Scott WE. Prediction of survival of critically ill patients by admission comorbidity. J Clin Epidemiol. 1996:49(7):743-7.

20. Fadem SZ. Charlson Comorbidity Scoring System: estimating prognosis for dialysis patients. http://touchcalc.com/calculators/cci_js. Accessed 16 Jun 2015.

21. Venkatram S, Chilimuri S, Adrish M, Salako A, Patel M, Diaz-Fuentes G. Vitamin $D$ deficiency is associated with mortality in the medical intensive care unit. Crit Care. 2011;15(6):R292.

22. Braun A, Chang D, Mahadevappa K, Gibbons FK, Liu Y, Giovannucci E, Christopher KB. Association of low serum 25-hydroxyvitamin D levels and mortality in the critically ill. Crit Care Med Apr. 2011;39(4):671-7.

23. Arnson Y, Gringauz I, Itzhaky D, Amital H. Vitamin D deficiency is associated with poor outcomes and increased mortality in severely ill patients. QJM. 2012;105(7):633-9.

24. Amrein K, Quraishi SA, Litonjua AA, Gibbons FK, Pieber TR, Camargo Jr CA, Giovannucci E, Christopher KB. Evidence for a U-shaped relationship between prehospital vitamin D status and mortality: a cohort study. J Clin Endocrinol Metab. 2014;99(4):1461-9.

25. Quraishi SA, Bittner EA, Blum L, McCarthy CM, Bhan I, Camargo Jr CA. Prospective study of vitamin $D$ status at initiation of care in critically ill surgical patients and risk of 90-day mortality. Crit Care Med Jun. 2014;42(6):1365-71.

26. Joshi A, Bhadade R, Varthakavi PK, DeSouza R, Bhagwat NM, Chadha MD. Vitamin $D$ deficiency is associated with increased mortality in critically ill patients especially in those requiring ventilatory support. Indian J Endocrinol Metab Jul. 2014;18(4):511-5.

27. Zajic P, Amrein K. Vitamin D deficiency in the ICU: a systematic review. Minerva Endocrinol. 2014:39(4):275-87.

28. Padhi R, Panda B, Jagati S, Patra SC. Vitamin D status in adult critically ill patients in Eastern India: an observational retrospective study. Lung India. 2014;31(3):212-6.

29. Braun AB, Gibbons FK, Litonjua AA, Giovannucci E, Christopher KB. Low serum 25-hydroxyvitamin $D$ at critical care initiation is associated with increased mortality. Crit Care Med Jan. 2012:40(1):63-72.

30. Quraishi SA, Camargo Jr CA. Vitamin D in acute stress and critical illness. Curr Opin Clin Nutr Metab Care. 2012;15(6):625-34.

31. Quraishi SA, McCarthy C, Blum L, Cobb JP, Camargo CA, Jr. Plasma 25hydroxyvitamin $D$ levels at initiation of care and duration of mechanical ventilation in critically ill surgical patients. JPEN J. Parenter. Enteral Nutr. 2015.

32. Cleveland WS. Robust locally weighted regression and smoothing scatterplots. Journal of the American Statistical Association. 1979;74(368):829-36.

33. Cleveland WS, Devlin SJ. Locally weighted regression: an approach to regression analysis by local fitting. Journal of the American Statistical Association. 1988;83(403):596-610.

34. Quraishi SA, Litonjua AA, Elias KM, Gibbons FK, Giovannucci E, Camargo Ir CA, Christopher KB. Association between pre-hospital vitamin D status and hospital-acquired new-onset delirium. Br J Nutr. 2015;113(11): 1753-60.

35. Quraishi SA, Litonjua AA, Moromizato T, Gibbons FK, Camargo Jr CA, Giovannucci E, Christopher KB. Association between prehospital vitamin D status and hospital-acquired Clostridium difficile infections. JPEN J Parenter Enteral Nutr Jan. 2015;39(1):47-55.

36. Quraishi SA, Litonjua AA, Moromizato T, Gibbons FK, Camargo Jr CA, Giovannucci E, Christopher KB. Association between prehospital vitamin D status and hospital-acquired bloodstream infections. Am J Clin Nutr. 2013; 98(4):952-9.
37. Cleves MA. From the help desk: comparing areas under receiver operating characteristic curves from two or more probit or logit models. The Stata Journal. 2002;2(3):301-13.

38. Ho KM, Dobb GJ, Knuiman M, Finn J, Lee KY, Webb SA. A comparison of admission and worst 24-hour Acute Physiology and Chronic Health Evaluation II scores in predicting hospital mortality: a retrospective cohort study. Crit Care. 2006;10(1):R4.

39. Capuzzo M, Valpondi V, Sgarbi A, Bortolazzi S, Pavoni V, Gilli G, Candini G, Gritti G, Alvisi R. Validation of severity scoring systems SAPS II and APACHE ॥ in a single-center population. Intensive Care Med. 2000;26(12):1779-85.

40. Shen JJ, Ginn GO. Financial position and adoption of electronic health records: a retrospective longitudinal study. J Health Care Finance Spring. 2012;38(3):61-77.

41. Quach S, Hennessy DA, Faris P, Fong A, Quan H, Doig C. A comparison between the APACHE II and Charlson Index Score for predicting hospital mortality in critically ill patients. BMC Health Serv Res. 2009;9:129.

42. Govani SM, Waljee AK, Stidham RW, Higgins PD. Increasing ultraviolet light exposure is associated with reduced mortality from Clostridium difficile infection. United European gastroenterology journal. 2015;3(2):208-14.

43. Quan H, Li B, Couris CM, Fushimi K, Graham P, Hider P, Januel JM Sundararajan V. Updating and validating the Charlson comorbidity index and score for risk adjustment in hospital discharge abstracts using data from 6 countries. Am J Epidemiol. 2011;173(6):676-82.

44. Christensen S, Johansen MB, Christiansen CF, Jensen R, Lemeshow S Comparison of Charlson comorbidity index with SAPS and APACHE scores for prediction of mortality following intensive care. Clin Epidemiol. 2011;3:203-11.

45. Moromizato T, Litonjua AA, Braun AB, Gibbons FK, Giovannucci E, Christopher KB. Association of low serum 25-hydroxyvitamin D levels and sepsis in the critically ill. Crit Care Med Jan. 2014:42(1):97-107.

46. Amrein K, Venkatesh B. Vitamin D and the critically ill patient. Curr Opin Clin Nutr Metab Care. 2012;15(2):188-93.

47. Maiya S, Sullivan I, Allgrove J, Yates R, Malone M, Brain C, Archer N, Mok Q, Daubeney P, Tulloh R, Burch M. Hypocalcaemia and vitamin D deficiency: an important, but preventable, cause of life-threatening infant heart failure. Heart. 2008:94(5):581-4.

48. Bouillon R, Carmeliet G, Verlinden L, van Etten E, Verstuyf A, Luderer HF, Lieben L, Mathieu C, Demay M. Vitamin D and human health: lessons from vitamin D receptor null mice. Endocr Rev. 2008;29(6):726-76.

49. Bischoff-Ferrari HA, Conzelmann M, Dick W, Theiler R, Stahelin HB. [Effect of vitamin $D$ on muscle strength and relevance in regard to osteoporosis prevention]. Z. Rheumatol. 2003;62(6):518-21.

50. Kankova M, Luini W, Pedrazzoni M, Riganti F, Sironi M, Bottazzi B, Mantovani A, Vecchi A. Impairment of cytokine production in mice fed a vitamin D3deficient diet. Immunology. 1991;73(4):466-71.

51. Pinheiro Da Silva F, Machado MC. Antimicrobial peptides: clinical relevance and therapeutic implications. Peptides. 2012;36(2):308-14.

52. Durr UH, Sudheendra US, Ramamoorthy A. LL-37, the only human member of the cathelicidin family of antimicrobial peptides. Biochim Biophys Acta. 2006;1758(9):1408-25.

53. Tollin M, Bergman P, Svenberg T, Jornvall H, Gudmundsson GH, Agerberth B. Antimicrobial peptides in the first line defence of human colon mucosa. Peptides. 2003;24(4):523-30.

54. Fabri M, Stenger S, Shin DM, Yuk JM, Liu PT, Realegeno S, Lee HM, Krutzik SR, Schenk M, Sieling PA, Teles R, Montoya D, lyer SS, Bruns H, Lewinsohn DM, Hollis BW, Hewison M, Adams JS, Steinmeyer A, Zugel U, Cheng G, Jo EK, Bloom BR, Modlin RL. Vitamin D is required for IFN-gamma-mediated antimicrobial activity of human macrophages. Sci Transl Med. 2011;3(104): 104 ra102.

55. Quraishi SA, De Pascale G, Needleman JS, Nakazawa H, Kaneki M, Bajwa EK, Camargo Jr CA, Bhan I. Effect of cholecalciferol supplementation on vitamin D status and cathelicidin levels in sepsis: a randomized placebo-controlled trial. Crit Care Med Sep. 2015;43(9):1928-37.

56. Asakura H, Aoshima K, Suga Y, Yamazaki M, Morishita E, Saito M, Miyamoto K, Nakao S. Beneficial effect of the active form of vitamin D3 against LPSinduced DIC but not against tissue-factor-induced DIC in rat models. Thromb Haemost. 2001;85(2):287-90.

57. Moller S, Laigaard F, Olgaard K, Hemmingsen C. Effect of 1,25-dihydroxyvitamin D3 in experimental sepsis. Int J Med Sci. 2007:4(4):190-5.

58. Horiuchi $H$, Nagata I, Komoriya K. Protective effect of vitamin D3 analogues on endotoxin shock in mice. Agents Actions. 1991;33(3-4):343-8. 
59. Vitamin D. Metabolites quality assurance program. 2010. http://www.nist. $\mathrm{gov} / \mathrm{mml} / \mathrm{csd} / \mathrm{vitdqap} . \mathrm{cfm}$. Accessed 30 May 2016.

60. Tai SS, Bedner M, Phinney KW. Development of a candidate reference measurement procedure for the determination of 25-hydroxyvitamin D3 and 25-hydroxyvitamin D2 in human serum using isotope-dilution liquid chromatography-tandem mass spectrometry. Anal Chem. 2010;82(5):1942-8.

61. Stepman HC, Vanderroost A, Van Uytfanghe K, Thienpont LM. Candidate reference measurement procedures for serum 25-hydroxyvitamin D3 and 25-hydroxyvitamin D2 by using isotope-dilution liquid chromatographytandem mass spectrometry. Clin Chem. 2011;57(3):441-8.

62. Stockl D, Sluss PM, Thienpont LM. Specifications for trueness and precision of a reference measurement system for serum/plasma 25-hydroxyvitamin D analysis. Clin Chim Acta. 2009;408(1-2):8-13.

63. Lee S, Oncescu V, Mancuso M, Mehta S, Erickson D. A smartphone platform for the quantification of vitamin D levels. Lab on a chip. 2014;14(8):1437-42.

64. The Fast Pack System 2013. www.qualigeninc.com. Accessed 30 May 2016.

65. Point of Care Diagnostics 2015. http://www.nanospeed.ca/. Accessed 30 May 2016.

Submit your next manuscript to BioMed Central and we will help you at every step:

- We accept pre-submission inquiries

- Our selector tool helps you to find the most relevant journal

- We provide round the clock customer support

- Convenient online submission

- Thorough peer review

- Inclusion in PubMed and all major indexing services

- Maximum visibility for your research

Submit your manuscript at www.biomedcentral.com/submit
Biomed Central 